\title{
Improved Multi Model Procedure to Explore Medical Image Retrieval based on Visual Semantic Signatures
}

\author{
${ }^{1}$ Shaik. Jakeer Hussain, ${ }^{2}$ Dr. R. Kiran Kumar \\ 1 Research Scholar, Department of Computer Science, Krishna University, Machilipatnam, A.P., India \\ 2 Assistant Professor, Department of Computer Science, Krishna University, Machilipatnam, A.P., India \\ Email id: jakeerhussainsk@gmail.com ${ }^{1}$ kirankreddi@gmail.com ${ }^{2}$
}

\begin{abstract}
In medical sectors, medical imaging is animportant concept in real time environments. Different types of medical images are captured and stored in digital format in medical research centers. Facing this type of large volume of image data with different types of image modalities, it is very important to implement efficient content based image retrieval (CBIR) for medical research centers. Visual features based image label indexing is a limitation to explore efficient image retrieval from different medical sources. So that, in this paper, we propose Novel Multi Model Semantic Approach (NMMSA) is introduced based on recent and advanced machine learning and visual based indexing approaches. In this approach, we first investigate the semantic analysis to integrate visual features based label of image from different medical image sources to provide connection to retrieve images based on label indexing with visual features. Experimental results with high amount of medical images have been shown the performance of the proposed approach in terms of medical image indexing and image retrieval systems.
\end{abstract}

Keywords: Image indexing, content based image retrieval, multi model, probabilistic semantic analysis and machine learning.

\section{INTRODUCTION}

The issue of finding comparative medical pictures in a large volume of image data store in light of substance is called Content Based Image Retrieval (CBIR) [1]. The conventional Text Based Image Retrieval (TBIR) approach has numerous pragmatic impediments [2] like the medical pictures in the accumulation being clarified physically, which turns out to be more troublesome as the extent of the medical picture gathering increments. Another essential confinement is the insufficiency in speaking to the medical picture content. CBIR approaches are proposed to defeat the impediments of content based medical picture recovery. Content Based Image Retrieval systems are very much looked into. Be that as it may, they are constrained by the quality and amount of the printed comments of the pictures. Powerful and productive substance based methodologies can be utilized related to content based strategies to enhance the precision and culmination of the query items. Propelled by the essential potential clinical advantages, content-based medical image recovery (CBMIR) has turned into an exceptionally dynamic research territory in the course of the most recent decade. Among many cutting edge strategies of CBMIR look into i.e. Novel Unsupervised Label Indexing (NULI), a standout amongst the most encouraging headings is to relate multimodal data (e.g., content and picture) or to utilize the blends of printed and visual investigation systems for more successful and proficient CBMIR.

In this paper, we center around two modalities that are generally accessible in genuine facility hone: visual data of a restorative picture and the comparing content comment of the therapeutic picture. One of the significant hindrances we have distinguished is that the semantic hole exists between the low-level highlights (eg, low-level visual and literary highlights) and the abnormal state therapeutic ideas. The way to tending to these issues is to grow new semantic significant highlights from multimodal data to connect the semantic hole and to empower viable and effective CBMIR. Another test is that this present reality information are extremely uproarious and some methodology data (eg, content comment) might miss from input. To address the missing methodology issue, new calculations that can determine the missing methodology data from the 


\section{International Journal of Research in Advent Technology, Vol.7, No.5, May 2019 E-ISSN: 2321-9637 Available online at www.ijrat.org}

current known methodology (eg, getting missing content explanation from known visual substance) are required for making the framework usable in clinical practice.

The objective of our exploration in this paper is to create, assess, and show new picture and printed investigation procedures for versatile semantic therapeutic picture content examination and recovery. Keeping in mind the end goal to conquer the constraints presented in the past passage, our concentration in this paper is to produce semantic highlights. Our Novel Multi Model Semantic Approach (NMMSA) approach can possibly generously enhance the execution of medicinal picture recovery. It additionally can possibly be connected in clinical practice and medicinal services applications. As the name suggest that our proposed approach consist two label indexed models, i.e. probabilistic semantic analysis approach and machine learning model. Probabilistic semantic analysis approach is used to integrate visual and content information from medical images to connect gap in between visual features and content information. Machine learning approach defines modularity missing data from known features with different data relations. Experimental results with large quantity, real-world healthcare pictures have shown that our strategy is a good solution for the next-generation healthcare picture listing and recovery system.

\section{BACKGROUND WORK}

Pictures are omnipresent in biomedicine and the picture watchers assume a focal part in numerous parts of present day medicinal services. Huge measures of medicinal picture information are caught and recorded in computerized organize amid the day by day clinical rehearse, therapeutic research, and training (in 2009, more than 117,000 pictures for each day in the Geneva radiology division alone). Confronting such a remarkable volume of picture information with heterogeneous picture modalities, it is important to build up a compelling and proficient restorative picture recovery framework for clinical practice and research. Customarily, medicinal picture recovery frameworks depend on content based recovery procedures that utilization the subtitles related with the pictures, also, frequently, the entrance is by persistent ID, as it were. Since the 1990s, we have seen expanding interests in content-based picture recovery for medicinal applications. One of the promising headings in contentbased medicinal picture recovery is to correspond multimodular data (e.g., content and picture data) to give better bits of knowledge. In this paper, we focus our endeavors on the best way to recover the most significant restorative pictures utilizing multi-modular data.

In particular, we utilize two modalities: the visual substance of the pictures (spoken to by visual highlights) and the printed data related with the pictures. The center thought for multi-modular recovery is established in data combination. Existing writing on multi-modular recovery can generally be characterized into two classes: include combination and recovery combination. The element combination methodology produces an incorporated component portrayal from different modalities. The recovery combination procedure alludes to the strategies that union the recovery results from various recovery calculations. Our proposed approach has a place with the principal classification (include combination) and is to a great extent propelled by Pham et al. [1] and Leinhart et al.[2]. In [1], the highlights from various modalities are standardized and connected to produce the component vectors. At that point, the Latent Semantic Analysis (LSA) is connected on these highlights for picture recovery. In [2], Lienhart et al propose a multi-layer likelihood Latent Semantic Analysis (NMMSA) to tackle the multi-modular picture recovery issue. Our proposed approach is not the same as Pham et al. [1] in that we don't just connect the highlights from various modalities. Rather, we speak to the highlights from various modalities as a multi-dimensional network and join these component vectors utilizing a broadened different model. Our strategy is additionally not quite the same as Lienhart et al. [2] since we utilize a solitary NMMSA display rather than various models. The significant commitment of our work is the new portrayal of a picture utilizing visual-printed "words". These "words" are produced from the visual descriptors and printed data utilizing the expanded numerous model. 


\section{International Journal of Research in Advent Technology, Vol.7, No.5, May 2019 E-ISSN: 2321-9637 Available online at www.ijrat.org}

\section{PROPOSED APPROACH}

We will present Multi Model Semantic Approach (NMMSA) approach in more detail. To the best of our insight, how to create compelling and effective semantic highlights for huge scale medicinal picture sets remains a testing and unsolved issue. In this paper, we will center around the advancement and assessment of new semantic examination methods by exploring and assessing imaginative visual and literary demonstrating and investigation strategies for creating the semantic highlights. These semantic highlights can possibly connect the semantic hole. In particular, we create two kinds of measurable realistic models that can meld the unmistakable modalities to produce the semantic highlights. The recently created semantic highlights are fit for catching this present reality therapeutic ideas successfully and proficiently. Besides, the proposed approach can deal with the missing methodology dependably. The semantic highlights are another portrayal space with the accompanying wanted highlights: (1) the comparability in this portrayal space suggests the likeness of the relating genuine therapeutic ideas and (2) the portrayal space can be produced dependably even in the circumstances where there are absent and uproarious modalities.

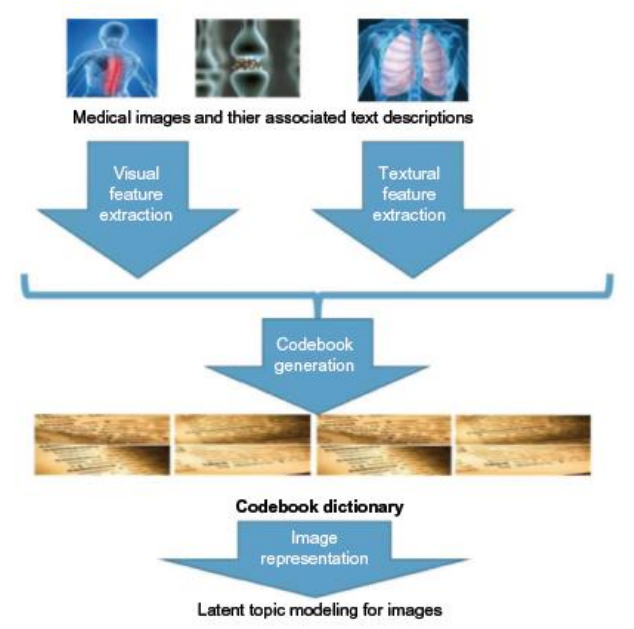

Figure 1. Basic procedure of proposed approach.

Figure 1 portrays an outline of the initial step. Our objective in this progression is to fabricate the realistic model and to create the inactive point portrayal for each picture in the database. Given the pictures and their related literary depictions, our calculations will create an idle theme portrayal for each picture. We utilize a stretched out NMMSA model to encode the visual what's more, printed data for each picture. The first NMMSA technique depends on a perspective model, which is a dormant variable show for general co-event information (eg, report word recurrence lattice). It shows the appropriation of words in the archive as a blend of a couple of perspectives. It was as of late utilized by the PC vision network to tackle the issues of picture recovery and question class acknowledgment. We broaden the NMMSA display by utilizing two irregular factors to speak to the visual and printed highlights, separately. If it's not too much trouble note, in our examination, we utilize the idea of visual bag ofwords (VBoW) model to extricate the underlying visual highlights from a picture. In this VBoW show, a picture is spoken to as a visual archive made out of visual components (a.k.a. visual words). This model has been extremely prominent over the most recent couple of years 55- 60 on account of its straightforwardness and versatility. In particular, we first apply scale-invariant feature transform (SIFT)- based intriguing point location methods 61 to recognize the potential remarkable focuses from the picture. For each fascinating point recognized by the SIFT strategy, we will extricate the SIFT descriptor, which is a 128dimensional vector. We at that point run k-implies bunching calculation for all the SIFT descriptors gathered from each preparation picture. The $\mathrm{k}$ centroid of the k-implies calculation are the visual words that can be utilized for late handling. For each picture, we look at the SIFT intriguing point and its SIFT descriptor with each visual word and discover the storeroom visual word. By along these lines, we can produce a histogram of visual words as a component portrayal for each picture.

Believe we have $\mathrm{D}\left(\mathrm{D}=\left\{\begin{array}{lll}\mathrm{d} 1 & \ldots \mathrm{dN}\end{array}\right)\right.$ pictures where di symbolizes the ith picture that contains both visible and textual details. We use two unique factors wv and wt to signify the visible and textual terms, respectively. We think that the visible terminology is showed as $\mathrm{WV}=\left\{\mathrm{WV} \_1, \ldots \mathrm{WV} \_\mathrm{M}\right\}$, while the textual terminology $\mathrm{WT}=\left\{\mathrm{wT}_{-} 1, \ldots \mathrm{wT} \_\mathrm{K}\right\}$. The corpus of 


\section{International Journal of Research in Advent Technology, Vol.7, No.5, May 2019 E-ISSN: 2321-9637 Available online at www.ijrat.org}

the picture data source can be described in a threedimensional co-occurrence matrix $\mathrm{N}$, whose level is $\mathrm{M}$ $\times \mathrm{K} \times \mathrm{N}$. The records $\mathrm{n}\left(\mathrm{wV} \_\mathrm{m}, \mathrm{wT} \_\mathrm{k}, \mathrm{dn}\right)$ in this matrix signify how often the phrase $w V_{-} m$ and $w_{-} T_{k}$ happened in picture dn.A hidden subject varying $\mathrm{z}$ is used to affiliate the existence of terms $\mathrm{wV}$ and $\mathrm{wT}$ to picture $\mathrm{d}$. The combined possibility design overWV $\times$ WT $\times \mathrm{D}$ is presented by the following equation:

$$
P\left(w_{V}, w_{T}, d\right)=P(d) P\left(w_{V}, w_{T} \mid d\right)
$$

After importing latent for further importing with different variables developed for multiple data dimensions

$$
P\left(z \mid w_{V}, w_{T}, d\right)=\frac{P(z) P(d \mid z) P\left(w_{V}, w_{T} \mid z\right) \text { th }}{\sum_{x \in Z} P\left(z^{\prime}\right) P\left(d \mid z^{\prime}\right) P\left(w_{V}, w_{T} \mid z^{\prime}\right)}
$$

Multi feature representation with different text labels from different medical images as follows:

$$
P(z) \alpha \sum_{d \in D} \sum_{w_{v} \in W_{v}} \sum_{w_{t} \in W_{T}} n\left(w_{v}, w_{t}, d\right) P\left(z \mid w_{v}, w_{t}, d\right)
$$

Our suggested extended NMMSA design,compared with the existing pLSA design, utilizes a threedimensionarray. Therefore, in comparison with the unique pLSAmodel, the variety of factors to be approximated during themachine learning methods is also improved. However, as indicated by above equations, the growing of factors will not cause the calculations intractable. Lastly, we use a histogram intersection (or possibly other range measures) to evaluate the similarity between the question picture and pictures in the database.

Once the two models (expanded NMMSA demonstrate appeared in Fig. 1 ) are prepared, we will speak to the visual and literary data utilizing the prepared models. In particular, we will decide the dispersion of the visual- literary words over the inert theme produced from the new NMMSA show. We will likewise produce the missing information utilizing contingent dissemination over the watched information.
Range analytics such as the histogram junction areemployed to estimate the likeness between the question imageand the data source pictures. Lastly, the

To get the visual highlights, we utilize a BoW model.63,69 Printed highlights are separated from the content comments related with the pictures. We apply the current vector-space model to the literary explanations. Some important pre-preparing (eg, evacuating stop words and stemming) is performed. Presently, each picture is spoken to by a twodimensional network, which shows the co-event of the visual- printed words in this picture. Accordingly, a three-dimensional framework speaks to the whole preparing information. At that point we apply the expectation-maximization (EM) calculation to this three-dimensional co-event table and get the model parameters. The last advance is to perform recovery. The objective is to register the likeness score between data source pictures areranked based on the likeness ranking.

\section{EXPERIMENTAL EVALUATION}

\section{a. Implementation:}

We will present the informational indexes utilized in our tests (segment Data sets), the rundown of execution measurements (area List of definitions for execution measurements), and in addition the itemized usage and trial results (segment System execution and point by point results). In the segment Data sets, we present the qualities of two informational collections:

ImageCLEF 2009 medicinal recovery test and Image-CLEF 2013 therapeutic recovery challenge. At that point we present the rundown of meanings of execution measurements at the area List of definitions for execution measurements. Some example measurements, for example, exactness, review, mean normal accuracy and precision (MAP), and ret_ret, utilized in our investigations are presented in this segment. At long last, we present our framework execution and present the exploratory outcomes with point by point examination in the area System execution and itemized results.

Data sets: We utilize two informational collections that have been generally utilized in therapeutic picture 


\section{International Journal of Research in Advent Technology, Vol.7, No.5, May 2019 E-ISSN: 2321-9637 \\ Available online at www.ijrat.org}

recovery research system. Using this implementation procedure on different medical images then visual representation of image as shown in figure 2 .

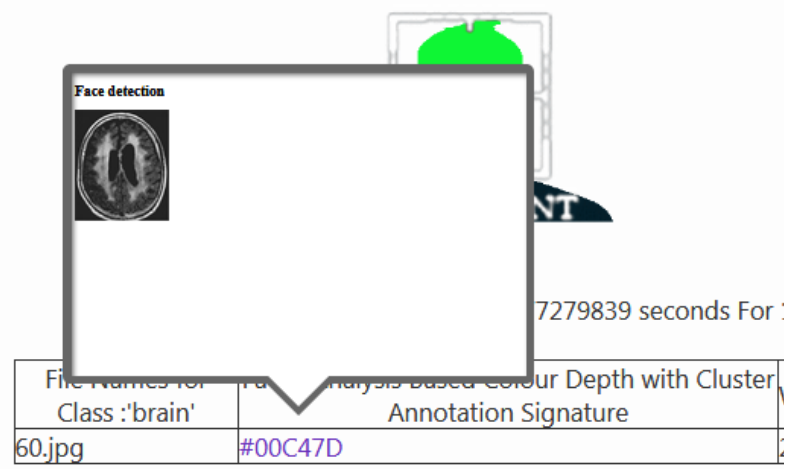

Figure 2. Implementation procedure of visual representation of image with color code representation.

Lastly, the rel_ret evaluate is actually another name for the variety of appropriate records in the recovered records according to the floor truth of different medical image sources.

\section{b. Results}

Our trial assessment, mainly evaluate with traditional strategy i.e Novel Unsupervised Label Indexing (NULI)and proposed approach (NMMSA) in terms of precision in marked medical picture recovery, perfection, remember, picture annotation with brand performance and time performance. Accuracy, Precision and Recall computation from different picture resources may strongly as follows:

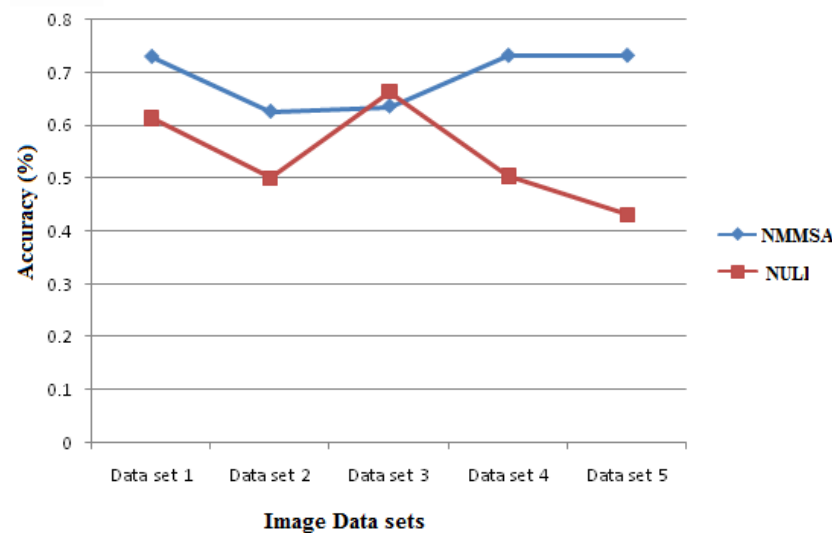

Figure 3. Performance of Accuracy with different attributes.

In picture recovery programs, perfection is the main parameter to understand more about the performance of the suggested strategy with evaluation of traditional techniques. Precision principles of both suggested and current strategy shown in figure 4 .

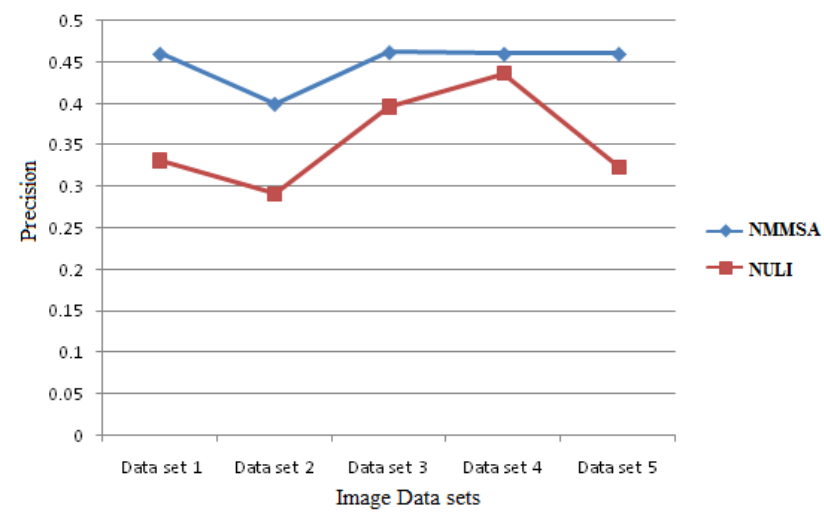

Figure 4. Performance of precision with medical image retrieval from different sources.

In picture recovery programs, remember is a referrals parameter to discover the performance of the suggested approach in terms of visual features based class references shown in figure 5 . 


\section{International Journal of Research in Advent Technology, Vol.7, No.5, May 2019 E-ISSN: 2321-9637 \\ Available online at www.ijrat.org}

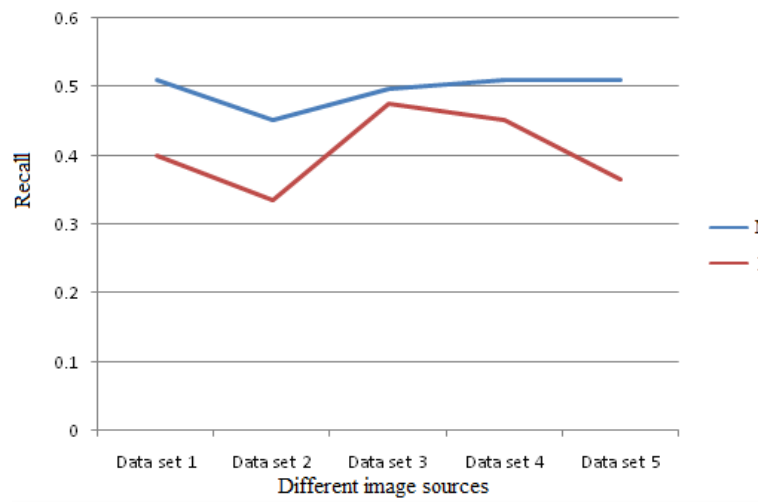

Figure 5. Performance of recall with different medical image sources.

A shown in the above figures, picture multiple picture recovery gives less efficiency to recover the topmost pictures for automated picture annotation with their titles and visual features from the medical picture databases, where as our strategy gives better accuracy and better performance. This means that our strategy is really a lot better compared to the traditional techniques with different picture features in visual features based medical picture annotation

\section{CONCLUSION}

Our proposed approach is an effective and efficient content based medical image retrieval for different medical research applications. This is very basic approach used to import different image label indexes for medical image sources. Different types of existing medical image indexing approaches were developed to retrieve efficient medical image retrieval but they are not supported to explore medical image relates to visual features of medical images. We developed a new multimodal medical picture recovery strategy based on the latest developments in mathematical visual design and strong learning. The trial results have proven that the suggested strategy is a good solution for nextgeneration healthcare picture listing and recovery system. In the future, we strategy to improve our suggested strategy with larger data places and to include healthcare ontology into our strategy. We also plan to discover the chance of developing our suggested strategy into medical practice.

\section{REFERENCES}

[1] Marcia A, Kassirer P, Relman A. Looking back on the Millennium in medicine. $\mathrm{N}$ Engl $\mathrm{J}$ Med. 2000;342:42-9.

[2] Diagnostic imaging market to increase to $\$ 26.6$ billion by 2016 by Companies \& Markets, a leading global aggregator of business information. 2013. Available at: http://www.companiesandmarkets.com/News/Healt hcare-and-Medical/Diagnostic-imaging-market-toincrease-to-26-6-billion-by-2016/NI6386.

[3] Roobottom C, Mitchell G, Morgan-Hughes G. Radiation-reduction strategies in cardiac computed tomographic angiography. Clin Radiol. 2010;65(11):859-67.

[4] AT\&T, Accenture Service Stores Medical Images in Cloud. Available at: http://www.informationweek.com/news/healthcare/ interoperability/232200581.

[5] Morin RL. Transforming the radiological interpretation process (TRIP). J Digital Imaging. 2004;17(2):78-9.

[6] Medical imaging in cancer care: charting the progress, prepared by Polidais LLC for Philips Healthcare. Available at: http://www.healthcare.philips.com/pwc_hc/us_en/a bout/Reimbursement/assets/docs/cancer_white_pap er.pdf.

[7] Brinkley JF, Greenes RA. Imaging and structural informatics. In: Shortliffe EH, Cimino JJ, eds. Biomedical Informatics: Computer Applications in Health Care and Biomedicine. 3rd ed. New York: Springer; 2006:344-78.

[8] Cao et al. Medical Image Retrieval: AMultimodal Approach. Cancer Informatics 2014:13(S3) 125136 doi: 10.4137/CIN.S14053.

[9] Müller H, Michoux N, Bandon D, Geissbuhler A. A review of content-based image retrieval systems in medicine - clinical benefits and future directions. Int J Med Inform. 2004;73:1-23.

[10] Lehmann TM, Güld MO, Thies C, et al. Contentbased image retrieval in medical applications. Methods Inf Med. 2004;43(4):354-61.

[11]Ruiz ME. Combining image features, case descriptions and UMLS concepts to improve retrieval of medical images. Paper presented at: Proceedings of American Medical Informatics Association Annual Symposium; 2006.

[12] Deserno TM, Antani S, Long R. Exploring access to scientific literature using content-based image retrieval. Paper presented at: Proceedings of the SPIE, Medical Imaging 2007: PACS and Imaging Informatics; 2007; San Diego, California, USA. 


\section{International Journal of Research in Advent Technology, Vol.7, No.5, May 2019 E-ISSN: 2321-9637 \\ Available online at www.ijrat.org}

[13] Müller H, Kalpathy-Cramer J. Analyzing the content out of context - features and gaps in medical image retrieval. J Healthcare Inf Sys Inf. 2009;4(1):88-98.

[14] Névéol A, Deserno TM, Darmoni SJ, Güld MO, Aronson AR. Natural language processing versus content-based image analysis for medical document retrieval. $J$ Am Soc Inf Sci Technol. 2009;60(1):123-34.

[15] Kalpathy-Cramer J, Hersh W. Medical image retrieval and automatic annotation: OHSU at ImageCLEF 2007. Paper presented at: Proceedings of 8th Workshop of the Cross-Language Evaluation Forum, CLEF; 2007; Budapest, Hungary.

[16] Lindberg DA, Humphreys BL, McCray AT. The unified medical language system. Methods Inf Med. 1993;32(4):281-91.

[17] Demner-Fushman D, Antani S, Siadat M-R, Soltanian-Zadeh H, Fotouhi F, Elisevich AK. Automatically finding images for clinical decision support. Paper presented at: Proceedings of the Seventh IEEE International Conference on Data Mining (ICDM) Workshops; 2007; Omaha, Nebraska, USA.

[18] Atmosukarto I, Travillian R, Franklin J, et al. A unifying framework for combining content-based image retrieval with relational database queries for biomedical applications. Paper presented at: Proceedings of Annual Meeting of the Society for Imaging Informatics in Medicine; 2008.

[19] Syeda-Mahmood T, Wang F, Beymer D, Amir A, Richmond M, Hashmi S. AALIM: Multi-modal Mining for healthcare decision support. Paper presented at: Proceedings of IEEE Conference on Computers in Cardiology (CinC), 2007-2009; Durham, North Carolina, USA.

[20] Müller H, Geissbuhler A. Medical Multimedia Retrieval 2.0 In: Year book of Medical Informatics - Methods of Information in Medicine. Murray P, Ed. Stuttgart, Germany: Schattauer Publishers. 2008;3:55-64.

[21]Chenna Reddy, P \& Giridhar Akula, V.S. \& Gangadhar, Yarabham. (2019). Adaptive digital medical image watermarking approach in 2Dwavelet domain using speed-up robust feature method. International Journal of Biomedical Engineering and Technology. 29. 273. 10.1504/IJBET.2019.10018576.

[22] Cao Y, Kalpathy-Cramer J, Ünay D. Medical multimedia analysis and retrieval. Paper presented at: Proceedings of the 19th ACM international conference on Multimedia (ACM MM 2011); 2011; Phoenix, AZ, U.S.A.
[23] Müller H, Greenspan H. Overview of the Third Workshop on Medical Content - Based Retrieval for Clinical Decision Support (MCBR-CDS 2012). Medical Content-Based Retrieval for Clinical Decision Support. Berlin: Springer; 2012:1-9.

[24] Benois-Pineau J, Briassouli A, Hauptmann A. ACM MM MIIRH 2013: workshop on multimedia indexing and information retrieval for healthcare. Paper presented at: Proceedings of the 21st ACM international conference on Multimedia; 2013.

[25] Pham T-T, Maillot NE, Lim J-H, Chevallet J-P. Latent semantic fusion model for image retrieval and annotation. Paper presented at: Proceedings of the sixteenth ACM conference on Conference on information and knowledge management (CIKM); 2007; Lisbon, Portugal 